DOI: https://doi.org/10.46296/ig.v4i8.0024

\title{
EMISIONES DE GASES CONTAMINANTES EN VEHÍCULOS LIVIANOS A GASOLINA
}

\section{EMISSIONS OF POLLUTING GASES IN LIGHT GASOLINE VEHICLES}

\author{
Velepucha-Sánchez Jorge Milton ${ }^{1}$; Sabando-Piguabe Luis Felipe ${ }^{2}$ \\ ${ }^{1}$ Estudiante de la Maestría en Mantenimiento Industrial, Mención Gestión Eficiente del \\ Mantenimiento, Instituto de Posgrado, Universidad Técnica de Manabí. Portoviejo, \\ Ecuador. jorge.velepucha@utm.edu.ec, ORCID ID: https://orcid.org/0000-0002-3600- \\ 5896
}

${ }^{2}$ Docente de la Carrera de Mecánica de la Facultad de Ciencias Matemáticas, Físicas

y Químicas, Universidad Técnica de Manabí, Portoviejo, Ecuador.

luis.sabando@utm.edu.ec, ORCID ID: https://orcid.org/0000-0001-7492-7472

\begin{abstract}
Resumen
El estudio tuvo como objetivo central la determinación de emisiones de gases contaminantes en vehículos livianos a gasolina del parque automotor de la universidad técnica de Manabí, Portoviejo, Ecuador. Como metodología se aplicó en enfoque cuantitativo de la investigación, mediante un diseño cuasi experimental, empleando la técnica de la observación apoyada en instrumentos tecnológicos para la toma de datos del objeto de estudio, tal como el analizador de gases, para posteriormente procesar la información en gráficas y tablas; la muestra poblacional la constituyeron 10 vehículos a gasolina, de los cuales 8 corresponden a la década del 2000 en adelante y 2 a la década del 90, según la normativa NTE INEN 2204. Se obtuvo como resultados los tipos de emisiones contaminantes producidos por los vehículos de ambas décadas, durante la prueba estática y la prueba dinámica, logrando establecerse el cumplimiento de los parámetros de emisiones contaminantes que norma la NTE INEN 2204, exceptuando los datos de un solo vehículo ( $1 \%$ de la muestra) que no cumplía la normativa, cuya fabricación corresponde a los años 90 . Se pudo concluir que los valores resultantes son producto de las condiciones del motor de los vehículos y su vida útil alargada dado el mantenimiento que se les proporciona en los centros de mantenimiento.
\end{abstract}

Palabras clave: contaminación, gases, gasolina, mantenimiento, vehículo.

\begin{abstract}
The main objective of the study was to determine the emissions of polluting gases in light gasoline vehicles of the automotive fleet of the Technical University of Manabí, Portoviejo, Ecuador. As a methodology, a quantitative approach to the research was applied, through a quasi-experimental design, using the technique of observation supported by technological instruments to collect data from the object of study, such as the gas analyzer, to later process the information in graphs and tables; The population sample was made up of 10 gasoline vehicles, of which 8 correspond to the 2000s onwards and 2 to the 90s, according to the NTE INEN 2204 regulation. The types of polluting emissions produced by the vehicles were obtained as results. of both decades, during the static test and the dynamic test, achieving compliance with the pollutant emissions parameters established by the NTE INEN 2204, except for the data of a single vehicle ( $1 \%$ of the sample) that did not comply with the regulations, whose manufacture corresponds to the $90 \mathrm{~s}$. It was possible to conclude that the resulting values are the product of the conditions of the vehicle's engine and its extended useful life given the maintenance provided in the maintenance centers.
\end{abstract}

Keywords: pollution, gases, gasoline, maintenance, vehicle.

Información del manuscrito:

Fecha de recepción: 03 de mayo de 2021.

Fecha de aceptación: 08 de junio de 2021.

Fecha de publicación: 09 de julio de 2021. 


\section{Introducción}

El desarrollo de las sociedades modernas se encuentra íntimamente ligado a una creciente presión sobre el medio ambiente, principalmente por la explotación y uso de los recursos naturales y energéticos, el incremento de la población, el transporte, o el uso de nuevas técnicas para obtener mayores rendimientos, estando también el destino de los residuos que se generan como consecuencia de su uso y consumo en la generación de efluentes residuales de tipo líquido, sólido y gaseoso (Parra, 2004), así varios reportes sobre la contaminación atmosférica evidencian la gravedad de este problema en las grandes ciudades (Hilario, 2017), datos de la Organización Mundial de la Salud (OMS, 2005) sobre la calidad del aire revelan contenidos de varios gases y material particulado que expone al $92 \%$ de la población mundial viviendo en lugares donde la calidad del aire excede los límites permisibles establecidos por la OMS.

La operación del transporte terrestre es precisamente una de las principales causas de los impactos negativos que se producen al medio ambiente; tales efectos son las emisiones contaminantes, el ruido, la basura, y los accidentes de tránsito, entre otros, generando que el mantenimiento vehicular sea una obligación ciudadana que asegure el rendimiento del para que su quema sea eficiente, propendiendo a la disminución de las emisiones de sustancias contaminantes hacia la atmósfera (Curi, 2019).

Uno de los medios de transporte terrestre que generan contaminación son los automóviles, éstos despiden contaminantes por el tubo de escape como consecuencia de la combustión de la gasolina y a través del capó y del sistema de combustible cuando el calor ocasiona la evaporación del mismo, la emisión por evaporación da lugar a diferentes situaciones como el efecto que la temperatura exterior en días cálidos y soleados provoca sobre el combustible haciendo que se evapore; cuando el calor del motor y del sistema de escape genera un aumento de la temperatura del combustible; cuando el motor se encuentra apagado y permanece lo suficientemente caliente ocasiona también la evaporación del 
combustible; es importante recordar que al cargar combustible en el vehículo, los vapores de la gasolina escapan del tanque a través del pico, por lo cual es recomendable cargar de combustible en las primeras horas de la mañana (Pérez D. , 2017).

Estas emisiones contaminantes de los automotores se generan por diversos procesos, una de las más frecuentes son las producidas por la quema del combustible y liberadas por el tubo de escape, los contaminantes más peligrosos incluyen al monóxido de carbono (CO); óxidos de nitrógeno (NOx); hidrocarburos ( $\mathrm{HC})$, óxidos de azufre (SOx); compuestos orgánicos volátiles (COV) y partículas en suspensión (PM), además de los gases de efecto invernadero como el dióxido de carbono (CO2), que no afectan la salud humana pero inciden en el cambio climático (Núnez, 2018). Además de las emisiones de COV por combustión, están las emisiones evaporativas ocasionadas por la evaporación del combustible cuando el automotor está en marcha 0 estacionado (Blancarte, 2011).
En Ecuador, estudios como el de Llanes, Rocha, Peralta, \& Leguisamo (2018) refieren el uso de diversos tipos de gasolina para la alimentación de vehículos, en función de su número de octano como la denominada extra (87 octanos), la gasolina súper equivalente al combustible Premium con 93 octanos y la eco país, un biocombustible a base de gasolina extra y $5 \%$ de bioetanol obtenido de la caña de azúcar, cuyas propiedades se asemejan a la gasolina extra y es comercializado en ciudades situadas a pocos metros sobre el nivel del mar.

Los tipos de combustible comercializados en el país, son generadores de contaminantes a la atmósfera, debido a los gases expulsados por el motor después de la combustión, en ese sentido, Pérez (2018) en su estudio, establece que es necesario realizar una comparación de los gases contaminantes ocasionados por combustibles como la gasolina súper, extra y eco-país, determinando, las normativas establecidas para el control de contaminantes, mediante la comparación de datos, en base a 
una caracterización del combustible, dada por el calor desprendido, teniendo en cuenta la principal fuente de emisión de $\mathrm{CO}_{2}$ que es la combustión causada por el uso de combustibles fósiles, gas carbón y petróleo; para poder determinar el combustible que menos contamina.

Según (Caiza \& Portilla, 2010) el cálculo de las emisiones contaminantes de las distintas fuentes móviles que circulan en zonas urbanas, es la base para una adecuada definición de políticas para la planificación del transporte y medidas de control de la contaminación, siendo necesario realizar evaluaciones cada cierto periodo, debido a que la tecnología de los vehículos cambia continuamente, por tanto, la cantidad de contaminantes emitidos por el proceso de combustión.

La investigación de (Lozano, Daza, Clavijo, \& Mantilla, 2018) revela que del total de vehículos que circulan por carreteras ecuatorianas, tales como automóviles, camiones y autobuses, el $95 \%$ son automotores de uso particular, los de alquiler representan el $3,3 \%$ los que pertenecen al Estado el $2 \%$ y los de uso Municipal el 0,4\%, algunos de estos cuentan con métodos obsoletos que ocasionan la combustión incompleta, lo que desencadena en una excesiva emisión de gases contaminantes, que afectan de forma directa en la salud de las personas principalmente a los niños y personas de la tercera edad.

La norma técnica ecuatoriana NTE INEN 2204 determina los límites permitidos de emisiones producidas por fuentes móviles terrestres a gasolina, en base a la cual, gases como el monóxido de carbono (CO) e hidrocarburos (HC) son los más contaminantes, siendo el monóxido de carbono el más peligroso de todos, ya que no se puede distinguir ni olfatear, formándose cuando la mezcla de combustible es rica y hay poco oxígeno para quemar completamente todo el combustible (Antamba, Reyes, \& Granja, 2016).

En tal sentido, la mayoría de los vehículos a gasolina actualmente en uso en el país, están equipados con catalizadores para el control de $\mathrm{CO}$, HC y NOx, los cuales son impactados por los niveles de azufre del combustible. El impacto del azufre es más severo conforme los 
vehículos están diseñados para alcanzar estándares más estrictos, los niveles actuales de azufre en el combustible son el obstáculo primario para introducir tecnologías más avanzadas de control de emisiones en el mercado, estas tecnologías

reducirían

dramáticamente los contaminantes convencionales e incluso promoverían el diseño de motores más eficientes en el uso del combustible (Blumberg, Walsh, \& Pera, 2003).

No obstante, aunque las normativas de emisiones de gases de escape y de consumo en el tráfico vial de todo el mundo son cada vez más exigentes, propendiendo al uso de combustibles más amigables con el medio ambiente, los vehículos a gasolina seguirán siendo demandados y su producción no decrece, así, entre más grande es el automotor, el consumo de combustible es mayor, ya que su motor de combustión seguirá siendo la tecnología de propulsión más importante del vehículo, pues las alternativas amigables como la propulsión eléctrica, aún necesitan muchos años de investigación y desarrollo $(\mathrm{I}+\mathrm{D})$ para llegar a ser económicamente viables en los grandes mercados (Sara, 2013).

El objetivo del presente trabajo se centra en determinar las emisiones contaminantes de vehículos livianos a gasolina del parque automotor de la Universidad Técnica de Manabí.

\section{Materiales y métodos}

\section{Localización}

La presente investigación se la desarrolló en el laboratorio de Máquinas Térmicas de la Universidad Técnica de Manabí, ubicada en la ciudad de Portoviejo, Manabí, Ecuador. En la fig. (2) siguiente se aprecia el lugar.

Figura 1. Localización del estudio.

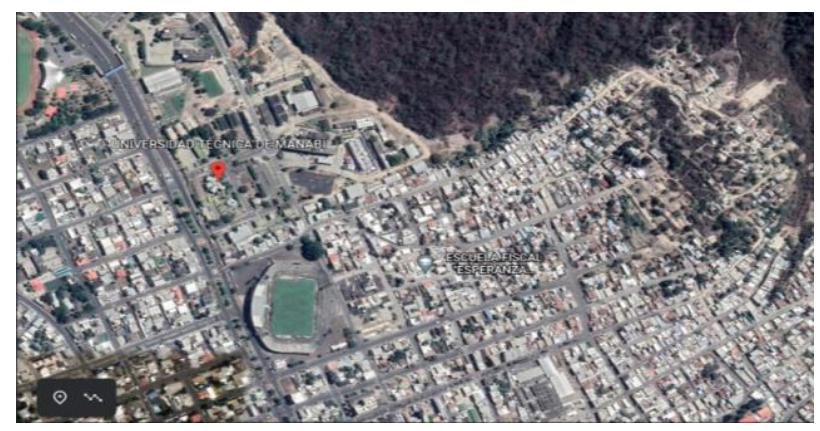

Fuente: Google Map (2021). 


\section{Población y muestra}

La tabla (1) siguiente muestra en detalle los 10 tipos de vehículos livianos a gasolina seleccionados para realizar el diagnóstico de emisiones de gases de los motores.

Tabla 1. Población y muestra.

\begin{tabular}{|l|l|l|}
\hline \multirow{2}{*}{$\#$} & \multicolumn{2}{|c|}{ Tipos de vehículos livianos a gasolina } \\
\cline { 2 - 3 } & Modelo 2000 y posteriores & Modelos 1990-1999 \\
\hline 1 & Aveo Emotion & Nissan Frontier \\
\hline 2 & BT50 Mazda & Toyota Caravan \\
\hline 3 & Sail Chevrolet & \\
\hline 4 & Aveo Activo & \\
\hline 5 & Nissan Sentra Se & \\
\hline 6 & Kia Sportage & \\
\hline 7 & Chevrolet Spark & \\
\hline 8 & Aveo Family & 2 tipos \\
\hline Total & 8 tipos & \\
\hline
\end{tabular}

Fuente: Tomado de UTM (2021). Elaborado por Autores.

Enfoque y diseño de la investigación

El enfoque de la investigación es cuantitativo, ya que se recogieron los datos de las emisiones de gases contaminantes producidas por los motores de 10 tipos de vehículos livianos a gasolina, utilizando instrumentos de medición y análisis para este propósito. En tal virtud, este enfoque "utiliza la recopilación de información para comprobar las hipótesis mediante el uso de estrategias estadísticas basadas en la medición numérica, lo cual permitiría al investigador proponer patrones de comportamiento $y$ probar los diversos fundamentos teóricos que explicarían dichos patrones" (Hernández, et al., 2014).
El diseño de la investigación es experimental del tipo cuasi experimental, puesto que se trabajó con la toma de las emisiones de gases de los vehículos de la muestra seleccionada midiéndose los resultados obtenidos. Así este diseño se refiere a diseños de investigación experimentales "en los cuales los sujetos o grupos de sujetos de estudio no están asignados aleatoriamente. Los diseños cuasiexperimentales más usados siguen la misma lógica e involucran la comparación de los grupos de tratamiento y control como en las pruebas aleatorias" (Rossi \& Freeman, 1993). 
Se aplicó el método analítico para realizar el análisis e interpretación de los datos resultantes del diagnóstico de las emisiones de gases de los motores de vehículos livianos a gasolina.

\section{Diseño cuasi experimental}

El diseño concibió la aplicación de la Norma técnica ecuatoriana NTE INEN 2 204: 2002 para la determinación de emisiones y gases contaminantes de los vehículos livianos a gasolina, mediante el uso del equipo compacto de análisis de emisiones para vehículos a gasolina MGT5 Marca MAHA.

\section{Fuente móvil:}

Vehículos livianos a gasolina de varios modelos y años.

Año

1990-1999, 2000 y posteriores.

Modelos: Aveo emotion, BT50 Mazda, Sail Chevrolet, Aveo Activo, Nissan Sentra SE, Kia Sportage, Chevrolet Spark, Aveo Family.

\section{Ciclo}

Tiempo necesario para que el vehículo alcance la temperatura normal de operación en condiciones de marcha mínima o ralentí. Para las fuentes móviles equipadas con electroventilador, ciclo es el período que transcurre entre el encendido del ventilador del sistema de enfriamiento y el momento en que el ventilador se detiene.

\section{Ciclos de prueba}

Secuencia de operaciones estándar a las que es sometido un vehículo automotor o un motor, para determinar el nivel de emisiones que produce. Para los propósitos de esta norma, los ciclos que se aplican son los siguientes:

\section{Ciclo ECE + EUDC}

Ciclo de prueba dinámico establecido por la Unión Europea para los vehículos livianos y medianos, que utilizan gasolina. NTE INEN 2204 2017-01 2016-849 2

\section{Ciclo FTP-75}

Ciclo de prueba dinámico establecido por la Agencia de Protección del Medio Ambiente de los Estados Unidos (EPA), para los vehículos livianos y medianos, que utilizan gasolina.

\section{Dinamómetro}

Aparato utilizado para medir la potencia generada por un vehículo 
automotor o motor solo, a través de aplicaciones de velocidad y torque.

\section{Emisión de escape}

Descarga al aire de una o más sustancias en estado sólido, líquido, gaseoso o de alguna combinación de estos, proveniente del sistema de escape de una fuente móvil.

\section{Marcha mínima o ralentí}

Especificación de velocidad del motor establecida por el fabricante 0 ensamblador del vehículo, requerida para mantenerlo funcionando sin carga y en neutro (para cajas manuales) y en parqueo (para cajas automáticas). Cuando no se disponga de la especificación del fabricante 0 ensamblador del vehículo, la condición de marcha mínima o ralentí se establecerá en un máximo de 1100 r.p.m.

\section{Motor}

Fuente principal de poder de un vehículo automotor que convierte la energía de un combustible líquido o gaseoso en energía cinética.

\section{Peso bruto vehicular (PBV)}

Peso total del vehículo, definido como la suma total del peso en vacío (tara) más la carga técnicamente admisible declarada por el fabricante.

\section{Peso de vehículo en vacío (tara)}

Valor nominal del peso del vehículo, según lo indicado por el fabricante, incluyendo todo el equipo estándar que requiere para su funcionamiento normal (por ejemplo, extintor de fuego, herramientas, rueda de emergencia, etc.), además de refrigerante, aceites, el tanque de combustible con su capacidad a la mitad.

\section{Peso de referencia (PR)}

Peso del vehículo en marcha aumentado con un peso fijo de 120 $\mathrm{kg}$. El peso del vehículo en marcha será el correspondiente al peso total en vacío con todos los depósitos llenos, salvo el del combustible, que estará solo a la mitad de su capacidad, un juego de herramientas y la rueda de repuesto.

\section{Prueba dinámica}

Medición de emisiones que se realiza con el vehículo o motor sobre un dinamómetro, aplicando los ciclos de prueba descritos en esta norma. NTE INEN 2204 2017-01 2016-849 3. 
Temperatura normal de operación

Temperatura que alcanza el motor después de operar un mínimo de 10 minutos en marcha mínima (ralentí), o cuando en estas mismas condiciones, la temperatura del aceite en el cárter del motor alcance $75^{\circ} \mathrm{C}$ o más. En las fuentes móviles equipadas con electroventilador, esta condición es confirmada después de operar un ciclo.

\section{Vehículo automotor}

Vehículo de transporte terrestre, de carga o de pasajeros, que se utiliza en la vía pública, propulsado por su propia fuente motriz.

\section{Ámbito de aplicación:}

Gestión ambiental. Aire. Vehículos automotores. Límites permitidos de emisiones producidas por fuentes móviles terrestres de gasolina

\section{Límites máximos:}

Límites máximos de emisiones permitidos para fuentes móviles con motor de gasolina. Marcha mínima o ralentí (prueba estática).

\section{Condición 1:}

Toda fuente móvil con motor de gasolina, durante su funcionamiento en condición de marcha mínima o ralentí y a temperatura normal de operación, no debe emitir al aire monóxido de carbono (CO) e hidrocarburos $(\mathrm{HC})$ en cantidades superiores a las señaladas en la tabla (2) siguiente.

Tabla 2. Límites máximos de emisiones permitidos para fuentes móviles con motor a gasolina.

Marcha mínima o ralentí (prueba estática)

\begin{tabular}{|c|c|c|c|c|}
\hline \multirow{2}{*}{ Año modelo } & \multicolumn{2}{|c|}{ C C* $^{*}$} & \multicolumn{2}{c|}{ Ppm HC$^{*}$} \\
\cline { 2 - 5 } & $0-1500^{* *}$ & $1500-3000^{* *}$ & $0-1500^{* *}$ & $1500-300^{* *}$ \\
\hline 2000 y posteriores & 1,0 & 1,0 & 200 & 200 \\
\hline 1990 a 1999 & 3,5 & 4,5 & 650 & 750 \\
\hline 1989 y anteriores & 5,5 & 6,5 & 1000 & 1200 \\
\hline
\end{tabular}

Fuente: Tomado de INEN (2013)

\section{Condición 2:}

Toda fuente móvil con motor de gasolina en su marcha dinámica, no debe emitir al aire monóxido de carbono (CO), hidrocarburos $(\mathrm{HC})$, óxidos de nitrógeno (NOx) y emisiones evaporativas, en cantidades superiores a las 
señaladas en la tabla (3) a

continuación.

Tabla 3. Límites máximos de emisiones para fuentes móviles de gasolina (prueba dinámica)

\begin{tabular}{|c|c|c|c|c|c|c|c|}
\hline & Clase & $\begin{array}{c}\text { Peso de } \\
\text { referencia (PR) kg }\end{array}$ & $\begin{array}{c}\mathrm{CO} \\
\mathrm{g} / \mathrm{km}\end{array}$ & $\begin{array}{l}\mathrm{HC} \\
\mathrm{g} / \mathrm{km}\end{array}$ & $\begin{array}{l}\mathrm{HC}+ \\
\mathrm{NOx} \\
\mathrm{g} / \mathrm{km}\end{array}$ & NOx & $\begin{array}{l}\text { Ciclo de } \\
\text { prueba }\end{array}$ \\
\hline$M^{a}$ & - & Todas & 2,3 & 0,2 & - & 0,15 & \multirow{4}{*}{$\begin{array}{c}\text { ECE + EUDC } \\
\text { (también } \\
\text { conocido como } \\
\text { MVEG-A) }\end{array}$} \\
\hline \multirow{3}{*}{$\mathrm{N} 1^{\mathrm{b}}$} & 1 & $\mathrm{PR}<=1305$ & 2,3 & 0,2 & - & 0,15 & \\
\hline & II & $1350<\mathrm{PR}<=1760$ & 4,17 & 0,25 & - & 0,18 & \\
\hline & III & $1760<\mathrm{PR}$ & 5,22 & 0,29 & - & 0,21 & \\
\hline
\end{tabular}

Fuente: Tomado de INEN (2013)

\section{Resultados y discusión}

La muestra seleccionada de 10 vehículos se la ha clasificado en 8 inherentes a la década del 2000 en adelante, y 2 de los años 1990 1999, de conformidad con la clasificación que refiere la norma técnica NTE INEN 2 204: 2002.

En la tabla (4) puede apreciarse las características de los motores de vehículos a gasolina de la década del 2000 en adelante.

Tabla 4. Caracterización de los motores de vehículos livianos a gasolina años 2000 y posteriores

\begin{tabular}{|l|l|l|l|l|ll|}
\hline $\begin{array}{l}\text { Marca } \\
\text { vehículo de }\end{array}$ & $\begin{array}{l}\text { Tipo de } \\
\text { motor }\end{array}$ & Cilindraje & $\begin{array}{l}\text { Sistema de } \\
\text { inyección }\end{array}$ & Válvulas & \multicolumn{2}{|l|}{ Observaciones } \\
\hline $\begin{array}{l}\text { AVEO } \\
\text { EMOTION }\end{array}$ & $\begin{array}{l}4 \\
\text { cilindros }\end{array}$ & 1398 CC. & $\begin{array}{l}\text { Electrónica } \\
\text { multipunto }\end{array}$ & $\begin{array}{l}16 \\
\text { válvulas }\end{array}$ & $\begin{array}{l}\text { Aceleración } \\
\text { (año 2012) }\end{array}$ & mecánica, \\
\hline BT50 MAZDA & $\begin{array}{l}4 \\
\text { cilindros }\end{array}$ & 2199 CC. & $\begin{array}{l}\text { Electrónica } \\
\text { multipunto }\end{array}$ & $\begin{array}{l}12 \\
\text { válvulas }\end{array}$ & $\begin{array}{l}\text { Aceleración } \\
\text { (año 2008) }\end{array}$ & mecánica, \\
\hline $\begin{array}{l}\text { SAIL } \\
\text { CHEVROLET }\end{array}$ & $\begin{array}{l}4 \\
\text { cilindros }\end{array}$ & 1399 CC. & $\begin{array}{l}\text { Electrónica } \\
\text { multipunto }\end{array}$ & $\begin{array}{l}16 \\
\text { válvulas }\end{array}$ & $\begin{array}{l}\text { Aceleración } \\
\text { (año 2013) }\end{array}$ & electrónica, \\
\hline $\begin{array}{l}\text { AVEO } \\
\text { ACTIVO }\end{array}$ & $\begin{array}{l}4 \\
\text { cilindros }\end{array}$ & 1599 CC. & $\begin{array}{l}\text { Electrónica } \\
\text { multipunto }\end{array}$ & $\begin{array}{l}16 \\
\text { válvulas }\end{array}$ & $\begin{array}{l}\text { Aceleración } \\
\text { (año 2010) }\end{array}$ & mecánica, \\
\hline $\begin{array}{l}\text { NISSAN } \\
\text { SENTRA SE }\end{array}$ & $\begin{array}{l}4 \\
\text { cilindros }\end{array}$ & 1999 CC. & $\begin{array}{l}\text { Electrónica } \\
\text { multipunto }\end{array}$ & $\begin{array}{l}16 \\
\text { válvulas }\end{array}$ & $\begin{array}{l}\text { Aceleración } \\
\text { (año 2009) }\end{array}$ & mecánica, \\
\hline $\begin{array}{l}\text { KIA } \\
\text { SPORTAGE }\end{array}$ & $\begin{array}{l}4 \\
\text { cilindros }\end{array}$ & 1999 CC. & $\begin{array}{l}\text { Electrónica } \\
\text { multipunto }\end{array}$ & $\begin{array}{l}16 \\
\text { válvulas }\end{array}$ & $\begin{array}{l}\text { Aceleración } \\
\text { (año 2014) }\end{array}$ & mecánica, \\
\hline $\begin{array}{l}\text { CHEVROLET } \\
\text { SPARK }\end{array}$ & $\begin{array}{l}4 \\
\text { cilindros }\end{array}$ & 999 CC. & $\begin{array}{l}\text { Electrónica } \\
\text { multipunto }\end{array}$ & $\begin{array}{l}8 \\
\text { válvulas }\end{array}$ & $\begin{array}{l}\text { Aceleración } \\
\text { (año 2007) }\end{array}$ & mecánica, \\
\hline AVEO FAMILY & $\begin{array}{l}4 \\
\text { cilindros }\end{array}$ & 1598 CC. & $\begin{array}{l}\text { Electrónica } \\
\text { multipunto }\end{array}$ & $\begin{array}{l}8 \\
\text { válvulas }\end{array}$ & $\begin{array}{l}\text { Aceleración } \\
\text { (año 2011) }\end{array}$ & mecánica, \\
\hline
\end{tabular}

Fuente: Tomado de (UTM, 2021). Elaborado por Autores.

Según el contenido de la tabla anterior, los vehículos de la década del 2000 y posteriores poseen motor de 4 cilindros, sistema de inyección de electrónica multipunto, cilindraje entre $999-2199 \mathrm{cc}, 8,12$ y 16 
válvulas, aceleración mecánica y uno que tiene aceleración electrónica.
En la tabla (5) se detallan las características de los motores de vehículos a gasolina de la década del 90.

Tabla 5. Caracterización de los motores de vehículos livianos a gasolina años 1990-1999.

\begin{tabular}{|l|l|l|l|l|l|}
\hline $\begin{array}{l}\text { Marca de } \\
\text { vehículo }\end{array}$ & $\begin{array}{l}\text { Tipo de } \\
\text { motor }\end{array}$ & Cilindraje & $\begin{array}{l}\text { Sistema de } \\
\text { inyección }\end{array}$ & Válvulas & Observaciones \\
\hline $\begin{array}{l}\text { NISSAN } \\
\text { FRONTIER }\end{array}$ & $\begin{array}{l}4 \\
\text { cilindros }\end{array}$ & 2399 CC. & $\begin{array}{l}\text { Convencional } \\
\text { (Carburador). }\end{array}$ & $\begin{array}{l}12 \\
\text { válvulas }\end{array}$ & $\begin{array}{l}\text { Aceleración mecánica, (año } \\
1994)\end{array}$ \\
\hline $\begin{array}{l}\text { TOYOTA } \\
\text { Corolla }\end{array}$ & $\begin{array}{l}4 \text { cilindros } \\
\text { cha CC. }\end{array}$ & $\begin{array}{l}\text { Electrónica } \\
\text { multipunto }\end{array}$ & $\begin{array}{l}16 \\
\text { válvulas }\end{array}$ & $\begin{array}{l}\text { Aceleración mecánica con } \\
\text { un inyector extra para } \\
\text { encendido en frío, (año } \\
1996)\end{array}$ \\
\hline
\end{tabular}

Fuente: Tomado de (UTM, 2021). Elaborado por Autores.

De acuerdo a lo descrito en la tabla precedente, aquellos automotores de la década de los 90, también poseen motor de 4 cilindros, sistema de inyección de electrónica multipunto, cilindraje de 1599 y 2399 cc, tienen 12 y 16 válvulas, aceleración mecánica y mecánica con inyector extra para encendido en frío.
En la tabla (6) se presenta los resultados de la toma de emisiones de gases contaminantes a los vehículos de la muestra, cuyos modelos corresponden al año 2000 en adelante, en su marcha mínima o ralentí.

Tabla 6. Emisión de gases de vehículos modelo 2000 y posteriores, en marcha mínima o ralentí

\begin{tabular}{|l|l|l|l|l|l|l|}
\hline Modelo & $\begin{array}{l}\text { Monóxido de } \\
\text { carbono (CO) }\end{array}$ & $\begin{array}{l}\text { Dióxido de } \\
\text { carbono } \\
\left(\mathbf{C O}_{2}\right)\end{array}$ & $\begin{array}{l}\text { Hidro } \\
\text { carburos } \\
\text { (HC) }\end{array}$ & $\begin{array}{l}\text { Oxígeno } \\
\mathbf{( O )}\end{array}$ & Lambda & RPM \\
\hline $\begin{array}{l}\text { Aveo } \\
\text { Emotion }\end{array}$ & $0,01 \%$ Vol. & $13,50 \%$ Vol. & $11 \mathrm{ppm}$ & $0,50 \%$ Vol. & 1.025 & $\begin{array}{l}830 \\
\mathrm{~min}^{-1}\end{array}$ \\
\hline $\begin{array}{l}\text { BT50 } \\
\text { Mazda }\end{array}$ & $0,39 \%$ Vol. & $12,30 \%$ Vol. & $78 \mathrm{ppm}$ & $3,37 \%$ Vol. & 1.168 & $\begin{array}{l}770 \\
\mathrm{~min}^{-1}\end{array}$ \\
\hline $\begin{array}{l}\text { Sail } \\
\text { chevrolet }\end{array}$ & $0,10 \%$ Vol. & $8,40 \%$ Vol. & $54 \mathrm{ppm}$ & $8,18 \%$ Vol. & 1.662 & $\begin{array}{l}940 \\
\mathrm{~min}^{-1}\end{array}$ \\
\hline \begin{tabular}{l} 
Aveo activo \\
\hline $\begin{array}{l}\text { Nissan } \\
\text { sentra SE }\end{array}$
\end{tabular} & $0,08 \%$ Vol. & $13,80 \%$ Vol. & $14 \mathrm{ppm}$ & $0,58 \%$ Vol. & 1.026 & $\begin{array}{l}730 \\
\mathrm{~min}^{-1}\end{array}$ \\
\hline $\begin{array}{l}\text { KIA } \\
\text { Sportage }\end{array}$ & $0,10 \%$ Vol. & $13,40 \%$ Vol. & $62 \mathrm{ppm}$ & $0,72 \%$ Vol. & 1.020 & $\begin{array}{l}720 \\
\mathrm{~min}^{-1}\end{array}$ \\
\hline
\end{tabular}




\begin{tabular}{|l|l|l|l|l|l|l|}
\hline $\begin{array}{l}\text { Chevrolet } \\
\text { Spark }\end{array}$ & $0,15 \%$ Vol. & $10,10 \%$ Vol. & $18 \mathrm{ppm}$ & $0,52 \%$ Vol. & 900 & $\begin{array}{l}630 \\
\mathrm{~min}^{-1}\end{array}$ \\
\hline Aveo family & $0,18 \%$ Vol. & $9,10 \%$ Vol. & $16 \mathrm{ppm}$ & $0,52 \%$ Vol. & 910 & $\begin{array}{l}650 \\
\mathrm{~min}^{-1}\end{array}$ \\
\hline
\end{tabular}

Fuente: Datos tomados por Autores (2021).

La tabla (7) muestra los resultados de la toma de emisiones de gases a los vehículos de la muestra, cuyos modelos son de la década de 1990 1999, en su marcha mínima o ralentí.

Tabla 7. Emisión de gases de vehículos modelos 1990-1999, en marcha mínima o ralentí

\begin{tabular}{|l|l|l|l|l|l|l|}
\hline Modelo & $\begin{array}{l}\text { Monóxido } \\
\text { de carbono } \\
(\mathbf{C O})\end{array}$ & $\begin{array}{l}\text { Dióxido } \\
\text { de } \\
\text { carbono } \\
\left(\mathbf{C O}_{2}\right)\end{array}$ & $\begin{array}{l}\text { Hidro } \\
\text { carburos } \\
\mathbf{( H C )}\end{array}$ & $\begin{array}{l}\text { Oxígeno } \\
(\mathbf{O})\end{array}$ & Lambda & RPM \\
\hline $\begin{array}{l}\text { Nissan } \\
\text { frontier }\end{array}$ & $4,03 \%$ Vol. & $9,00 \%$ Vol. & $183 \mathrm{ppm}$ & $3,77 \%$ Vol. & 1.051 & $\begin{array}{l}950 \\
\mathrm{~min}^{-1}\end{array}$ \\
\hline $\begin{array}{l}\text { Toyota } \\
\text { caravan }\end{array}$ & $4,05 \%$ Vol. & $9,10 \%$ Vol. & $173 \mathrm{ppm}$ & $3,88 \%$ Vol. & 1.055 & $\begin{array}{l}980 \\
\mathrm{~min}^{-1}\end{array}$ \\
\hline
\end{tabular}

Fuente: Datos tomados por Autores (2021).

Como puede apreciarse en las tablas precedentes (6 y 7 ), los resultados de las emisiones de gases tomadas a los vehículos con motores a gasolina, cuyos modelos corresponden al año 2000 y posterior a ese año, arrojaron resultados dentro de los parámetros establecidos en la Norma Técnica Ecuatoriana NTE INEN 2 204: 2002. De la misma manera, aquellos vehículos cuyos modelos son de los años 1990 a 1999 también mostraron resultados acordes a la Norma Técnica Ecuatoriana NTE INEN 2 204: 2002. La referida norma establece "los límites permitidos de emisiones de contaminantes producidas por fuentes móviles terrestres (vehículos automotores) que emplean gasolina. Esta norma se aplica a las fuentes móviles terrestres de más de tres ruedas (vehículo automotor, vehículo prototipo)" (INEN, 2017).

Lo expuesto se debe a que el control de las emisiones de gases se efectuó cuando el vehículo estaba en marcha mínima o ralentí, ya que las revoluciones que presentan los vehículos livianos a gasolina en su marcha mínima están en un rango de 600 a $900 \mathrm{rpm}$. En la figura (2) se resumen los niveles de emisiones de $\mathrm{CO}$ y HC durante la prueba estática. 
Figura 2. Resumen de emisiones de CO y HC de la prueba estática aplicada a los automóviles analizados.

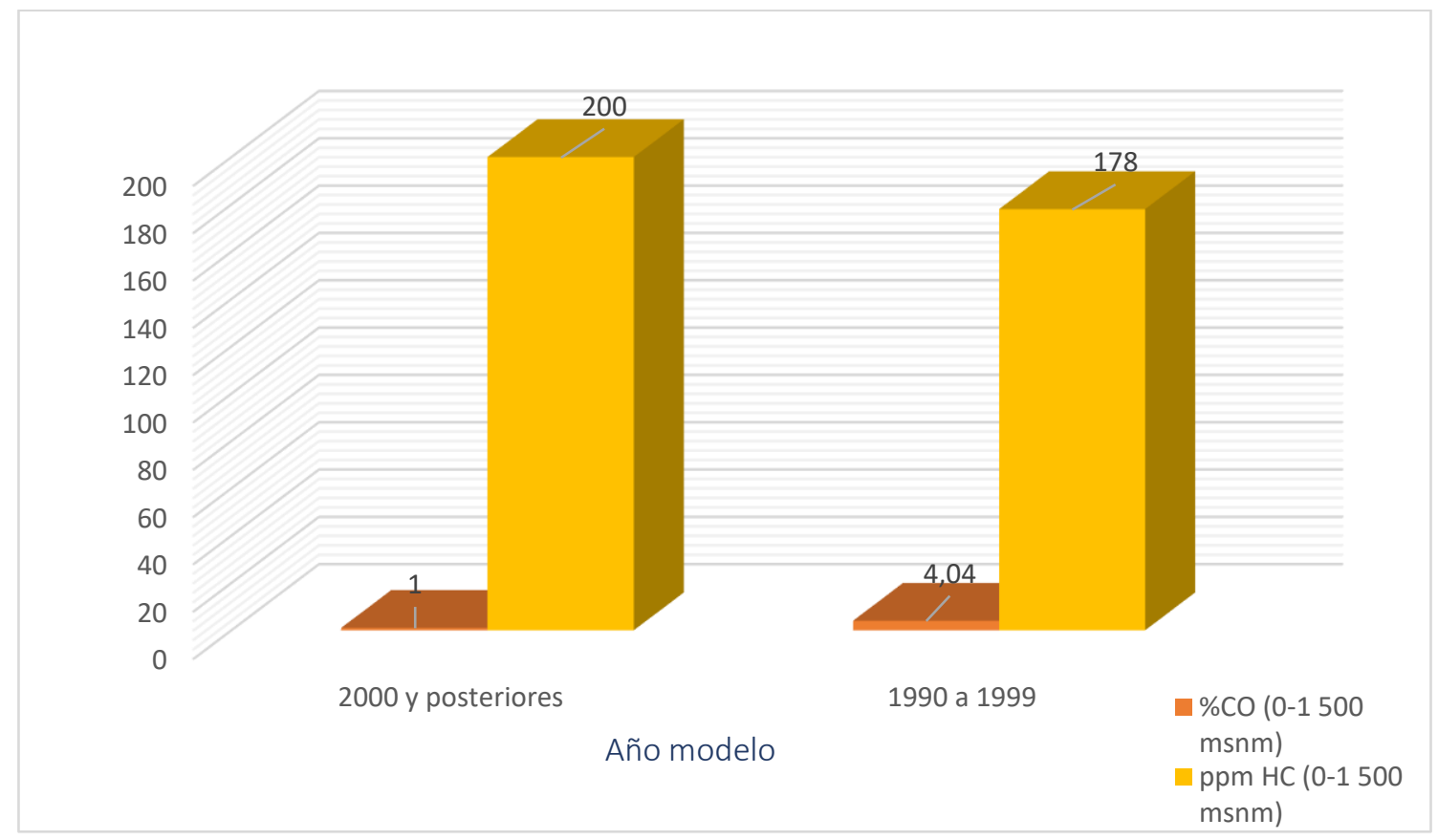

Fuente: Datos tomados por Autores (2021).

Teniendo en cuenta la altitud en metros sobre el nivel del mar (msnm) de la ciudad de Portoviejo, en donde se realizaron las pruebas a los vehículos livianos a gasolina; el volumen de las emisiones de monóxido de carbono (CO) de los automotores de la década de los 90 , se encuentran entre $4,03-4,05 \%$, que superan el límite máximo permitido por la NTE que es de $3,5 \%$. Las emisiones de hidrocarburos (HC) están en un rango entre 173183 ppm comparado con el límite máximo permisible por la NTE que es de 650 ppm, lo que significa que cumple con la normativa.
En los vehículos del año 2000 y posteriores, las emisiones de $\mathrm{CO}$ arrojaron un rango entre $0,01-0,41 \%$ que equivale al $1 \%$, cuyos resultados están por debajo del límite establecido en la norma técnica. Las emisiones de $\mathrm{HC}$ presentaron valores entre 11-78 ppm, que también se encuentran muy por debajo de los niveles de permisibilidad normada de 200 ppm.

En la tabla (8) se presenta los resultados de la toma de emisiones de gases a los vehículos de la muestra, cuyos modelos corresponden al año 2000 en adelante, en su marcha dinámica. 
Tabla 8. Emisión de gases de vehículos modelo 2000 y posteriores, en prueba dinámica.

\begin{tabular}{|c|c|c|c|c|c|c|}
\hline Modelo & $\begin{array}{l}\text { Monóxido } \\
\text { de } \\
\text { carbono } \\
\text { (CO) }\end{array}$ & $\begin{array}{l}\text { Dióxido } \\
\text { de } \\
\text { carbono } \\
\left(\mathrm{CO}_{2}\right)\end{array}$ & $\begin{array}{l}\text { Hidro } \\
\text { carburos } \\
\text { (HC) }\end{array}$ & $\begin{array}{l}\text { Oxígeno } \\
\text { (0) }\end{array}$ & Lambda & RPM \\
\hline Aveo Emotion & $1.5 \mathrm{~g} / \mathrm{Km}$ & $\begin{array}{l}13,50 \% \\
\text { Vol. }\end{array}$ & $0,12 \mathrm{~g} / \mathrm{Km}$ & $\begin{array}{l}1.14 \% \\
\text { Vol. }\end{array}$ & 1.058 & ${ }_{1}^{2580 \text { min }^{-}}$ \\
\hline BT50 Mazda & $1.65 \mathrm{~g} / \mathrm{Km}$ & $\begin{array}{ll}13,30 \% \\
\text { Vol. }\end{array}$ & $0.15 \mathrm{~g} / \mathrm{Km}$ & $\begin{array}{ll}0.83 \% \\
\text { Vol. }\end{array}$ & 1.034 & $\begin{array}{l}2220 \text { min }^{-} \\
1\end{array}$ \\
\hline Sail Chevrolet & $1.6 \mathrm{~g} / \mathrm{Km}$ & $\begin{array}{ll}8,30 & \% \\
\text { Vol. } & \\
\end{array}$ & $0.18 \mathrm{~g} / \mathrm{Km}$ & $\begin{array}{ll}8,03 & \% \\
\text { Vol. } & \\
\end{array}$ & 1.655 & $\begin{array}{l}2480 \text { min }^{-} \\
\end{array}$ \\
\hline Aveo activo & $0.12 \mathrm{~g} / \mathrm{Km}$ & $\begin{array}{ll}13,80 \% \\
\text { Vol. }\end{array}$ & $0.18 \mathrm{~g} / \mathrm{Km}$ & $\begin{array}{ll}0,35 \% \\
\text { Vol. }\end{array}$ & 1.016 & $\underbrace{2420 \text { min }^{-}}_{1}$ \\
\hline Nissan Sentra SE & $0.31 \mathrm{~g} / \mathrm{Km}$ & $\begin{array}{ll}13.60 \% \\
\text { Vol. }\end{array}$ & $0.11 \mathrm{~g} / \mathrm{Km}$ & $\begin{array}{ll}0.43 \% \\
\text { Vol. }\end{array}$ & 1.009 & $\begin{array}{l}2420 \text { min }^{-} \\
1\end{array}$ \\
\hline KIA Sportage & $0.50 \mathrm{~g} / \mathrm{Km}$ & $\begin{array}{l}12,80 \\
\text { Vol. }\end{array}$ & $0.15 \mathrm{~g} / \mathrm{Km}$ & $\begin{array}{ll}0,65 \% \\
\text { Vol. }\end{array}$ & 1.015 & $\begin{array}{l}2450 \text { min }^{-} \\
\end{array}$ \\
\hline Chevrolet Spark & $1.15 \mathrm{~g} / \mathrm{Km}$ & $\begin{array}{ll}11,10 \% \\
\text { Vol. }\end{array}$ & $0.18 \mathrm{~g} / \mathrm{Km}$ & $\begin{array}{ll}0,55 \% \\
\text { Vol. }\end{array}$ & 1095 & $\underbrace{2435 \text { min }^{-}}_{1}$ \\
\hline Aveo family & $1.8 \mathrm{~g} / \mathrm{Km}$ & $\begin{array}{l}9,15 \quad \% \\
\text { Vol. }\end{array}$ & $0.16 \mathrm{~g} / \mathrm{Km}$ & $\begin{array}{l}0,68 \% \\
\text { Vol. }\end{array}$ & 1010 & $\underbrace{2425 \text { min }^{-}}_{1}$ \\
\hline
\end{tabular}

Fuente: Datos tomados por Autores (2021).

La tabla (9) muestra los resultados de la toma de emisiones de gases a los vehículos de la muestra, cuyos modelos son de la década de 1990 1999, en su marcha dinámica.

Tabla 9. Emisión de gases de vehículos modelos 1990-1999, en prueba dinámica

\begin{tabular}{|l|l|l|l|l|l|l|}
\hline Modelo & $\begin{array}{l}\text { Monóxido } \\
\text { de carbono } \\
\text { (CO) }\end{array}$ & $\begin{array}{l}\text { Dióxido de } \\
\text { carbono } \\
\left(\mathbf{C O}_{2}\right)\end{array}$ & $\begin{array}{l}\text { Hidro } \\
\text { carburos } \\
\text { (HC) }\end{array}$ & $\begin{array}{l}\text { Oxígeno } \\
\mathbf{( O )}\end{array}$ & Lambda & RPM \\
\hline $\begin{array}{l}\text { Nissan } \\
\text { Frontier }\end{array}$ & $1.379 \mathrm{~g} / \mathrm{Km}$ & $9.20 \%$ Vol. & $0.185 \mathrm{~g} / \mathrm{Km}$ & $\begin{array}{l}3.77 \quad \% \\
\text { Vol. }\end{array}$ & 1.060 & $2670 \mathrm{~min}^{-1}$ \\
\hline $\begin{array}{l}\text { Toyota } \\
\text { caravan }\end{array}$ & $1.45 \mathrm{~g} / \mathrm{Km}$ & $9,15 \%$ Vol. & $0.173 \mathrm{ppm}$ & $\begin{array}{l}3,88 \% \\
\text { Vol. }\end{array}$ & 1.055 & $2480 \mathrm{~min}^{-1}$ \\
\hline
\end{tabular}

Fuente: Datos tomados por Autores (2021).

Como puede apreciarse en las tablas precedentes (8) y (9), los resultados de las emisiones de gases tomadas a los vehículos con motores a gasolina, cuyos modelos corresponden al año 2000 y posterior a ese año, arrojaron resultados dentro de los parámetros establecidos en la Norma Técnica Ecuatoriana NTE INEN 2 204: 2002 que establece los límites máximos de emisiones para fuentes móviles a gasolina en la prueba dinámica. De la misma manera, aquellos vehículos cuyos modelos son de los años 1990 a 1999 también mostraron resultados acordes a la Norma Técnica Ecuatoriana NTE INEN 2 204: 2002. La referida norma establece "Ios límites permitidos de 
emisiones de contaminantes producidas por fuentes móviles terrestres (vehículos automotores) que emplean gasolina. Esta norma se aplica a las fuentes móviles terrestres de más de tres ruedas (vehículo automotor, vehículo prototipo)" (INEN, 2017).

El análisis se lo efectuó con los vehículos en marcha dinámica, obteniendo tanto en los vehículos del año 2000 y posteriores, y en los de la década de los 90, valores dentro de emisiones de monóxido de carbono (CO) dentro del parámetro de 2.3 $\mathrm{g} / \mathrm{km}$ y de hidrocarburos (HC) hasta $0.2 \mathrm{~g} / \mathrm{km}$. No se analizaron las emisiones de óxidos de nitrógeno (NOx) que la norma los regula hasta 0.15. la figura (3) muestra el resumen de las emisiones de $\mathrm{CO}$ y $\mathrm{HC}$ durante la prueba dinámica.

Figura 3. Resumen de emisiones de CO y HC de la prueba dinámica aplicada a los automóviles analizados.

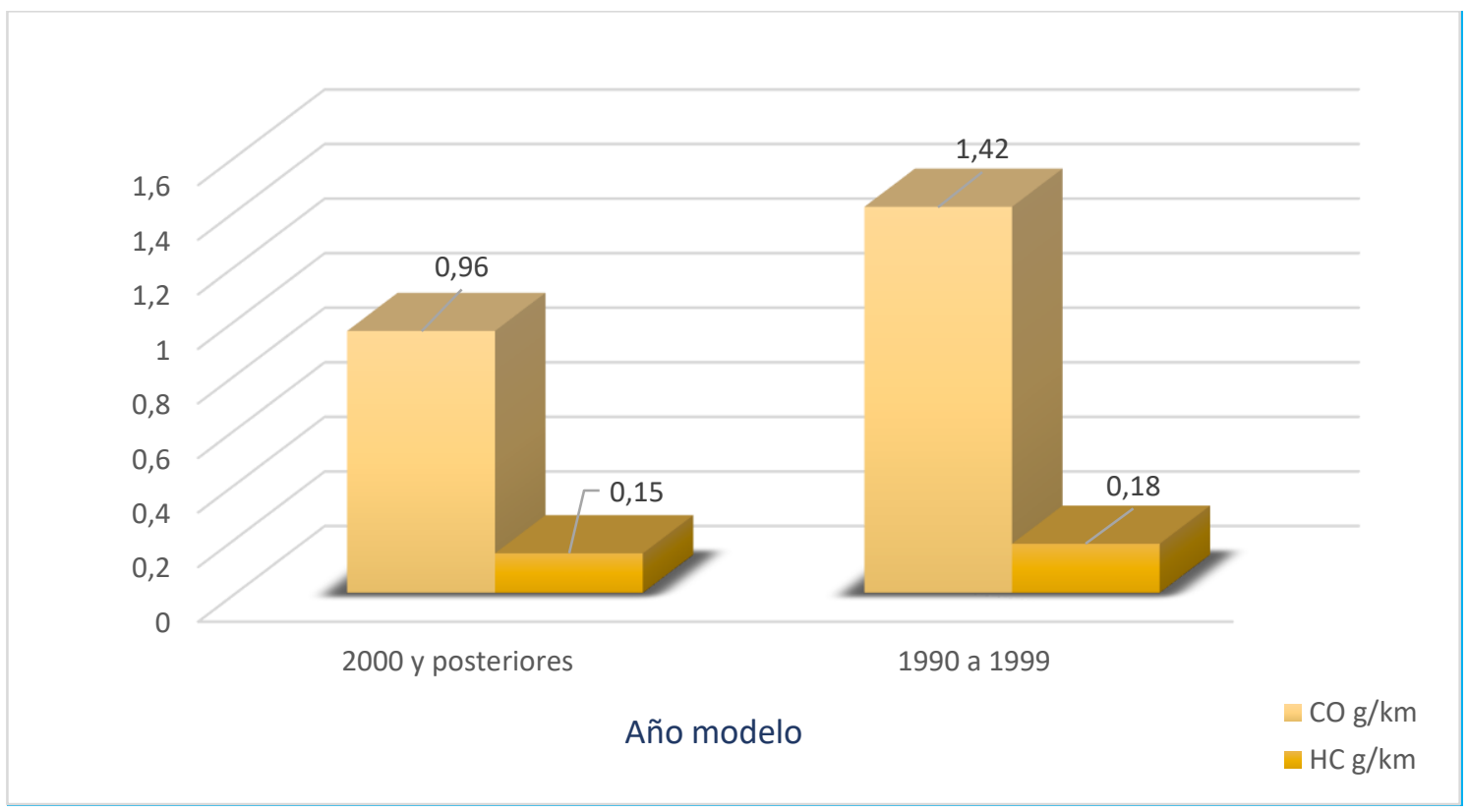

Fuente: Datos tomados por Autores (2021).

Teniendo en cuenta la altitud en metros sobre el nivel del mar (msnm) de la ciudad de Portoviejo, en donde se realizaron las pruebas a los vehículos livianos a gasolina; el volumen de las emisiones de monóxido de carbono (CO) de los automotores de la década de los 90 , se encuentran entre 1.38-1.45 g/km, que está dentro del límite máximo permitido por la NTE que es de 2.3 $\mathrm{g} / \mathrm{km}$. Las emisiones de hidrocarburos $(\mathrm{HC})$ están en un rango entre 0.17-0.19 $\mathrm{g} / \mathrm{km}$ comparado con el límite máximo permisible por la NTE que es de 0.2 
$\mathrm{g} / \mathrm{km}$, lo que significa que cumple con la normativa. En los vehículos del año 2000 y posteriores, las emisiones de $\mathrm{CO}$ arrojaron un rango entre $0.12-1.8 \mathrm{~g} / \mathrm{km}$ cuyos resultados están por debajo del límite establecido en la norma técnica (2.3 $\mathrm{g} / \mathrm{km})$. Las emisiones de $\mathrm{HC}$ presentaron valores entre 0.11-0.18 $\mathrm{g} / \mathrm{km}$, que también se encuentran muy por debajo de los niveles de permisibilidad normada de $0.2 \mathrm{~g} / \mathrm{km}$.

\section{Conclusiones}

Se logró caracterizar los vehículos livianos a gasolina para realizar los dos tipos de prueba establecidos en la norma técnica ecuatoriana NTE INEN 2 204: 2002, clasificándolas en automotores del año 1990 - 1999, y del año 2000 y posteriores. Los resultados de la prueba dinámica en todos los vehículos analizados, tanto de la década de los 90 y del 2000 en adelante, se encuentran dentro de los parámetros establecidos en la norma técnica, sin embargo, durante la prueba estática se obtuvieron valores fuera del rango especificado en la NTE correspondientes a los niveles de monóxido de carbono (CO) en $0,54 \%$ en los vehículos de los años 90, pudiendo concluir que, estos valores se originan por las condiciones del motor de los vehículos y su vida útil alargada dado el mantenimiento que se les proporciona.

\section{Bibliografía}

Antamba, J., Reyes, G., \& Granja, M. (2016). Estudio comparativo de gases contaminantes en un vehículo M1. Enfoque UTE, 110 -119. Obtenido de http://scielo.senescyt.gob.ec/ pdf/enfoqueute/v7n3/1390-

6542-enfoqueute-7-0300110.pdf

Blancarte, J. (2011). autocosmos. Obtenido de ¿Cuáles son los gases contaminantes más comunes que emiten los autos?:

https://noticias.autocosmos.c om.pe/2011/01/03/cuales-

son-los-gases-

contaminantes-mas-

comunes-que-emiten-losautos

Blumberg, K., Walsh, M., \& Pera, C. (2003). GASOLINA Y DIESEL DE BAJO AZUFRE: LA CLAVE PARA DISMINUIR LAS EMISIONES VEHICULARES. 1-78. Obtenido de https://theicct.org/sites/default /files/Bajo_Azufre_ICCT_200 3.pdf

Caiza, P., \& Portilla, A. (2010). DETERMINACIÓN DE LA 
INFLUENCIA DE LA ALTURA EN EMISIONES CONTAMINANTES DE UN VEHICULO CON MOTOR DE CICLO OTTO, DE INYECCION ELECTRÓNICA DE GASOLINA. Quito: Repositorio de la Escuela Politécnica Nacional. Obtenido de http://bibdigital.epn.edu.ec/ha ndle/15000/768

Curi, J. (2019). Estimación de las emisiones del co2 relacionado con el consumo de combustible y recorrido del servicio urbano de auto colectivo en la ciudad de cerro de Pasco 2017. Perú: Universidad Nacional Daniel Alcides Carrión.

Hernández, et al. (2014). Metodología de la Investigación. Barcelona: McGraw Hill.

Hilario, N. (2017). EMISIONES CONTAMINANTES DE VEHICULOS DEL DISTRITO DE HUANCAYO. Huancayo Perú: Universidad Nacional del Centro del Perú. Obtenido de

http://repositorio.uncp.edu.pe/ bitstream/handle/20.500.1289 4/4137/Hilario\%20Roman.pdf ?sequence $=1$ \&isAllowed $=y$

INEN. (2017). Norma Técnica Ecuatoriana NTE INEN 2 204: 2002. GESTIÓN

AMBIENTAL. AIRE. VEHÍCULOS AUTOMOTORES. LIIMITES
PERMITIDOS DE EMISIONES PRODUCIDAS POR FUENTE MÓVILES TERRESTRES QUE EMPLEAN GASOLINA. Quito, Pichincha, Ecuador: Insittuto Ecuatoriano de Normalización. Obtenido de https://www.normalizacion.go b.ec/buzon/normas/nte_inen 2204-2.pdf

Llanes, E., Rocha, J., Peralta, D., \& Leguisamo, J. (2018). Evaluación de emisiones de gases en un vehículo liviano a gasolina en condiciones de altura. Caso de estudio Quito, Ecuador. Enfoque UTE, 149158. Obtenido de http://scielo.senescyt.gob.ec/ pdf/enfoqueute/v9n2/13906542-enfoqueute-9-0200149.pdf

Lozano, C., Daza, O., Clavijo, M., \& Mantilla, C (2018). Determinación de los contaminantes mediante la opacidad, producto de la combustión por fuentes móviles a diésel en el cantón la Maná, provincia de Cotopaxi. Ciencia digital, 3151.

doi:https://doi.org/10.33262/ci enciadigital.v2i4.2..200

Núnez, C. (2018). Estudio de emisiones de gases en vehículos a gasolina. Ambato: Universidad Técnica de Ambato.

OMS. (2005). Guías de calidad del aire de la OMS relativas al 
material particulado, el ozono, el dióxido de nitrógeno y el óxido de azufre. Ginebra: Organización Mundial de la Salud. Obtenido de http://apps.who.int/iris/bitstrea $\mathrm{m} /$ handle/10665/69478/WHO _SDE_PHE_OEH_06.02_spa .pdf;jsessionid=3FA175AE31 10CF3EC9D117E2D779FB8 $\mathrm{C}$ ?sequence $=1$

Parra, R. (2004). Desarrollo del modelo EMICAT2000 para la estimación de emisiones de contaminantes del aire en Cataluña y su uso en modelos de disperción fotoquímica. Barcelona: Universidad de Catalunya. Obtenido de https://www.tesisenred.net/bit stream/handle/10803/6829/0 2Rrpn02de11.pdf?sequence $=$ 2\&isAllowed $=y$

Pérez, D. (2017). Estudio De Emisiones Contaminantes Utilizando Combustibles Locales. INNOVA Research Journal, 23-24. Obtenido de https://www.google.com/url?s $\mathrm{a}=\mathrm{t} \& \mathrm{rct}=\mathrm{j} \& \mathrm{q}=\&$ esrc $=$ s\&source $=$ web $\& c d=\& v e d=2 a h U K E$ win mZSp-

v7xAhUFQjABHVL5AyoQFjA MegQIHBAD\&url=https\%3A $\% 2 \mathrm{~F} \% 2 \mathrm{Fdialnet.unirioja.es \%}$ 2Fdescarga $\% 2$ Farticulo\%2F6 369767.pdf\&usg=AOvVaw1E ulRajy6_kTi5og7c6ewK

Pérez, D. (2018). Estudio de emisiones contaminantes utilizando combustibles locales. Revista de la
Universidad Internacional del Ecuador., 23-34. DOI: https://doi.org/10.33890/innov a.v3.n3.2018.635

Rossi, P., \& Freeman, H. (1993). Evaluation. A Systematic Approach, 5th Ed. California: SAGE.

Sara, S. (2013). Universidad Nebrija. Obtenido de Tecnologías para reducir el consumo y las emisiones:

https://www.nebrija.com/medi os/clubdelautomovil/2013/10/ 23/tecnologias-para-reducirel-consumo-y-las-emisiones/ 